\title{
Decoding Evolution of Native Fishes in Garhwal Himalaya using Molecular Markers and DNA Barcoding
}

\author{
Madhu Thapliyal, Bipin Sati, Ashish Thapliyal, K. K. Joshi
}

\begin{abstract}
As we are moving forward into the modern era of science, several new technologies have revolutionized various branches of science. Techniques of biodiversity conservation, fish biology etc. has also adapted to modern techniques. For a long time, most of the researches in taxonomy, including fisheries science were based on morphology and traditional methods. After the decade of 90's, slowly severalmolecular markers like RFLP, RAPD, SNP's etc. made inroad into taxonomy and fisheries. Molecular markers have several applications in the field of livestock improvement and understanding population dynamics to name a few. Since the 2004, a specific molecular marker, generally known as DNA Barcoding for species identification, came up. This molecular marker is a part of mitochondrial genome that encodes for Cytochrome C Oxidase Unit I (also called as COX or COI). It is advantageous because it has been tested across several animal species and it can differentiate species very well. This marker has also been used as a forensic tool to identify the species. In the current paper, we have used this molecular marker to decode evolution of native fishes of Garhwal Himalayan region. Over 350 barcodes were developed and these barcodes were used to for phylogenetic analysis.
\end{abstract}

Key words- Molecular Markers, DNA Bar-coding, Evolution, Himalaya, Breeding, livestock

\section{INTRODUCTION}

Himalaya has diverse and extensive network of fresh water rivers, streams, lakes etc. All these fresh water bodies harbor diverse aquatic fauna with fishes being the most extensively studied. There are many fish species reported by many authors in Uttarakhand. It is suggested that the native fish species of Himalayan region might be one of the earliest inhabitant of these fresh water systems and hence they are a good model to study evolution unfolding. These fishes have been well documented. However, prior to the year 2000, most of the studies were based on morphological characters and books like "Day Fauna" were served as the "KEY" for identification of fishes. All these so called "KEYS" were extensive illustrations of each species. About 2500 species of fishes have been reported in India and approximately 930 of these are fresh water fishes. The Himalayan region of India harbor's about 225 of these fresh water fishes. Various researchers have reported up to 50 different fish species from Garhwal Himalayan region.

Revised Manuscript Received on August 10, 2019.

*Madhu Thapliyal, Associate Professor and Head, Department of Zoology, Govt. PG College, Maldevta, Raipur, Dehradun, India.

Bipin Sati, USERC, Govt. of Uttarakhand, Dehradun, India.

Ashish Thapliyal, Professor, Department of Biotechnology, Graphic Era Deemed to be University, Dehradun, India.

K. K. Joshi, Department of Environment Science, Graphic Era Hill University, Dehradun, India.
*Corresponding Author

In Uttarakhand, most of the fresh water fishery resourcesare contributed by the River Yamuna or River Ganges. Fishes of Rivers Ganges (and is tributaries) has been well documented by fishes of River Yamuna in Garhwal Himalaya have not been well known except some contributions. There are numerous morphological based studies but there are only few report investigating fish species using molecular markers. Molecular markers are also being used used for assessing biodiversity using environmental DNA and meta-genomics (Krehenwinkel et. al., 2019; Adams et. al., 2019; Xing et. al. 2020). In about last three decades, the scenario of most of the Himalayan region as changed due to fast changing ecology of upland waters. Impact of anthropogenic activity on genome of fish species is among the interest in Himalayan region. The genetic variation in Himalayan region due to the regular floods in rivers and Dam constructions still not reported. Attempts have also been made to generate the DNA barcode $\&$ Population genetics of fishes, but most of the attempts are limited to major rivers i.e. the Ganges and the Yamuna (Thapliyal et al., 2013).

The molecular markers based on DNA are helpful to provide evolutionary relationship among different populations and cryptic species identification. The current paper is an attempt to investigate these changes using molecular markers specially focusing on DNA barcoding.

\section{WHY MOLECULAR MARKERS}

During several studies on morphological characters, research encountered a dilemma. There were several individuals which looked alike or had only small variations. A good example is that of Schizothorax species. It needs an expert to identify (ID) the two species of Schizothorax namely $S$. progastus and $S$. richardsonii and even after identification there could be queries about the ID. This happens in several species that they look alike but they are actually different species genetically. The latest example is of Giraffe (Petzold\&Hassanin, 2020). To solve this issue, molecular markers emerged and as they are specific sequences of DNA, these studies when coupled with morphometric studies were considered better option for species level identification. Introduction of molecular biology techniques in fisheries had a huge impact on the entire fishery research. Through application of these techniques we can figure out the variations in specific regions of genome. We can also develop a marker for desired characters and identify species based on DNA Barcode which is somewhat similar or just like a product barcode.

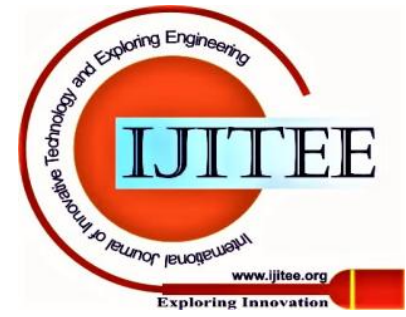




\section{MOLECULAR MARKERS}

Molecular marker is a specific sequence located on a specific gene. During the process of new progeny formation, inheritance of specific character occurs and these molecular markers stay together with the desired character. Hence the name molecular markers as the desired character can be followed by just following the molecular marker sequence. The interest in the DNA sequence based molecular markers had started as soon as the DNA model was presented by Watson and Crick and this model was awarded a Nobel Prize. This was followed by a rapid development of new technologies and methods like Polymerase Chain Reaction and DNA Sequencing. Once the sequencing of genome started, it opened an entire new ear of molecular markers and even a change in single nucleotide in a gene could be followed - technically called as Single Nucleotide Polymorphsim.

\section{WHY MITOCHONDRIAL MARKER:}

Besides the nuclear DNA, the eukaryotic mitochondria also has an extra DNA. This mitochondrial genome codes for 37 genes (two rRNAs, 22 tRNAs and 13 polypeptides).Mitochondrial DNA is of interest because of its unique features. These features are: a) This DNA is maternally inherited, b) it is a haploid molecule, c) there is no recombination process, d) there is no repair mechanism during DNA replication process e) there are several mitochondria in a cell and so it can be isolated and targeted easily, f) there are no introns in mitochondrial genome and g) mitochondrial genome is not too big and the optimum size makes it a favorite h) The COI marker can be used as a universal marker across entire animal species.The COI marker was first reported by Dr. Paul Hebert from University of Guelph, Canada as a molecular marker that can be used effectively to develop molecular database based catalogue of various animals inhabiting different regions (Hebert et al., 2003; Hebert et al., 2004). The sequences of these markers can also be compared using different available software's of sequence alignment and analysis.

\section{STUDIES IN GARHWAL HIMALAYAN REGION OF UTTARAKHAND :-}

As Garhwal Himalayan region is the origin point of River Ganges and River Yamuna, it is important to understand the evolutionary context of fauna inhabiting these river. Attempts have been made to generate the DNA barcode \&study population genetics of fishes in these rivers. Besides these river, there are several small tributaries that also inhabited by many species. In the entire study - one of the molecular marker called as COI - Cytochrome C Oxidase Unit I has been used. A specific region of this gene is sequenced and the bases are represented as colour codes and hence the name DNA Barcode (Fig. 1).

Example: GenBank Accession numbers NCBI ID JN965201

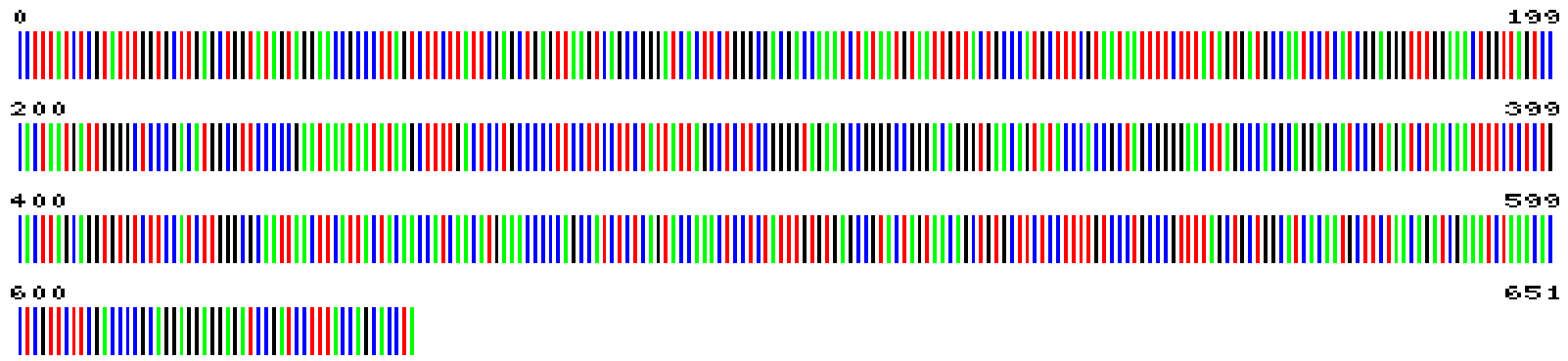

\footnotetext{
rTCGGGCTGAGCTAAGCCAACCTGGGTCGCTTCTTGGGGACGACCAAATCTATAATGTTAT

TGTTACTGCCCATGCTTTCGTAATAATTTTCTTTATAGTAATACCAATTCTTATTGGGGG GTTCGGAAACTGACTGGTACCCCTAATGATTGGAGCTCCCGATATAGCGTTCCCCCGAAT GAACAATATAAGCTTTTGACTCCTGCCCCCATCGTTCCTCTTGCTTCTAGCCTCATCTGG CGTGGAGGCCGGAGCCGGAACGGGGTGAACAGTTTACCCCCCACTGGCTGGAAACCTGGC CCATGCGGGAGCATCTGTAGACTTAACAATTTTCTCCCTTCACTTAGCGGGTGTATCATC CATCCTGGGAGCAATTAATTTTATTACTACAACCATCAACATAAAACCCCCAGCTATTTC TCAATATCAAACACCCTTATTCGTCTGAGCTGTTTTGGTTACAGCCGTACTTCTCCTTCT TTCCCTGCCTGTCCTAGCTGCTGGAATCACAATACTTCTTACAGACCGAAACCTCAATAC CTCATTTTTTGATCCTGCGGGAGGTGGGGACCCCATCCTTTACCAACACTTG
}

Fig 1.The concept of DNA Barcode. A sequence is converted to a barcode. 
Table 1 -Molecular markers and their description

\begin{tabular}{|c|c|c|}
\hline S.No. & Marker & Details \\
\hline 1. & $\begin{array}{l}\text { RFLP }(\text { Restriction } \\
\text { Fragment Length } \\
\text { Polymorphism) }\end{array}$ & $\begin{array}{l}\text { In this method, one or more restriction enzyme(s) are used to cut a DNA } \\
\text { isolated from the desired samples. The DNA digested by these restriction } \\
\text { enzymes is then run on a gel which give a unique banding pattern. These } \\
\text { patterns are used to a analysis called as RFLP (Restriction Fragment Length } \\
\text { Polymorphism). RFLP analysis is used in population studies (Ferguson et al., } \\
\text { 1995). }\end{array}$ \\
\hline 2. & $\begin{array}{c}\text { RAPD } \\
\text { (Random Amplification } \\
\text { of Polymorphic DNA) }\end{array}$ & $\begin{array}{l}\text { RAPD technique is a PCR based technique. Several primers are used on the } \\
\text { same DNA sample and then the amplified regions profile is developed. } \\
\text { (Hadryset al., 1992). }\end{array}$ \\
\hline 3. & Dloop region & $\begin{array}{l}\text { There is a region in mitochondrial region which is a non-coding region. This } \\
\text { region is called as The D-loop region. Variations of this region is mapped in } \\
\text { case of studies using D-loop as a marker. }\end{array}$ \\
\hline 4. & $\begin{array}{l}\text { VNTRs } \\
\text { (Variable number } \\
\text { tandem repeats) }\end{array}$ & $\begin{array}{l}\text { When the eukaryote genome was analyzed, it was surprising to note that there } \\
\text { were several unique segments of sequences that were repeated several times } \\
\text { (from } 10 \text { to } 100 \text { or more, O'Reilly and Wright, 1995). These repeated units can } \\
\text { be of two types - first one called as mini-satellite DNA ( } 9-65 \text { bp long), and } \\
\text { second called as microsatellite DNA ( } 4-8 \text { bp long). (Magoulas, 1998). }\end{array}$ \\
\hline 5. & COI & $\begin{array}{l}\text { The mitochondrial genome COI gene is an approximate } 656 \text { bp region. The } \\
\text { gene encodes part of the terminal enzyme of the respiratory chain of } \\
\text { mitochondria. }\end{array}$ \\
\hline 6. & Cytocrome b & $\begin{array}{l}\text { Cytochrome b is a component of electron transport gene and is used in some } \\
\text { studies. This gene is too long (about } 1,140 \mathrm{bp} \text { ) and sometimes the DNA } \\
\text { sequencing data of longer genes is cumbersome to handle. }\end{array}$ \\
\hline 7. & 16s rRNA & 16S rRNA gene has been used extensively for bacterial identification. \\
\hline 8. & ATPase $6 / 8$ & A region of mitochondrial ATPase gene \\
\hline
\end{tabular}

Table 2.Some of research papers in the Uttarakhand region on molecular marker.

\begin{tabular}{|c|c|c|c|c|}
\hline Species & References & Marker used & Year & Place/Area \\
\hline DawkinsiaTambraparniei & karuppiahkannan & Cytocrome b & 2014 & Uttarakhand \\
\hline Labeogonius & GrishmaTewari. & RAPD & 2013 & Uttarakhand \\
\hline $\begin{array}{l}\text { S. richardsonii, T. putitora, B. } \\
\text { Bendelisis and G. Gotyla.Danio }\end{array}$ & Thapliyal M & $\mathrm{COI}$ & 2013 & Uttarakhand \\
\hline $\begin{array}{l}\text { S. richardsonii, T. putitora, } B . \\
\text { Bendelisis and G. Gotyla }\end{array}$ & HimaniPandey. & 16SRNA & 2013 & Uttarakhand \\
\hline Bariliusbendelisis & A. K. Mishra. & RAPD & 2012 & Uttarakhand \\
\hline S. richardsonii and S. progastus. & SureshChandra. & COI & 2012 & Uttarakhand \\
\hline $\begin{array}{c}\text { Golden Mahseer Tor } \\
\text { putitora, Snow trout, Schizothorax } \\
\text { richardsonii, Indian trout, Raiamus } \\
\text { bola Garra, Garragotyla }\end{array}$ & G.K.Sivaraman. & $\begin{array}{l}\text { RAPD, } 12 S \\
\text { rRNA }\end{array}$ & 2012 & Uttarakhand \\
\hline Bariliusbendelisis & SeemaSah. & cytocrome b & 2011 & Uttarakhand \\
\hline Schizothorax richardsonii & Ashoktaru Barat. & cytocrome b & 2011 & Uttarakhand \\
\hline Eutropiichthysvacha & Gyan Chandra. & RAPD & 2010 & Uttarakhand \\
\hline Tor putitora & Mamta Singh. & $45 \mathrm{~S}$ and $5 \mathrm{~S}$ & 2009 & Uttarakhand \\
\hline
\end{tabular}

\section{MATERIAL AND METHODS}

Study site: The present study was a 200 kilometre radius of Garhwal Himalaya (30N; 78E approx.) Uttarakhand. Sampling sites included Bakot to Ponta Sahib in River Yamuna and from Bhatwari to Rishikesh in river Ganges. 


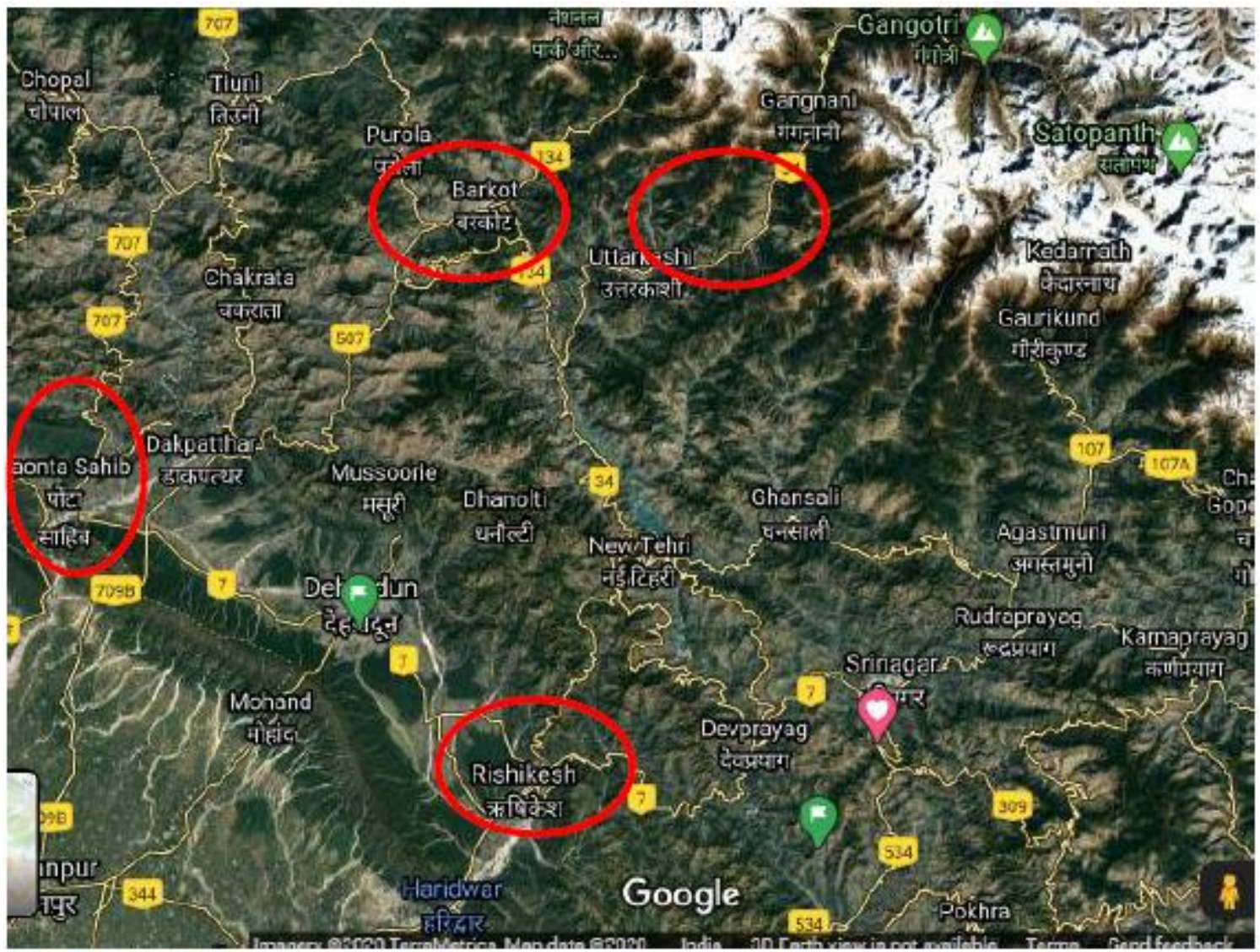

DNA extraction, PCR amplification, and sequencing.

The DNA was isolated from fish fin (Wizard Genomic DNA Purification Kit, Cat\# A1120, Qiagen Integrity). The isolated DNAs was then checked on 1\% Agarose gel and quantified (Nanodrop 1000 spectrophotometer, Applied bio system). Method used was as per Thapliyal et. al., 2013. In short, Samples were subjected to PCR using universal primers (FF2d (forward): TTCTCCACCAACCACAARGAYATYGGFR, FF1d (reverse): CACCTCAGGGTGTCCGAARAAYCARAA) The thermal cycler program was initial Denaturation at $95^{\circ} \mathrm{C}$ for $5 \mathrm{~min}$ followed by 35 cycles of $95^{\circ} \mathrm{C}$ for $30 \mathrm{sec}$ of Denaturation, $55^{\circ} \mathrm{C}$ of annealing for $30 \mathrm{sec}$ and $72^{\circ} \mathrm{C}$ of extension for $1 \mathrm{~min}$ and final extension of $72^{\circ} \mathrm{C}$ for $7 \mathrm{~min}$ and then the samples were stored at $4^{0} \mathrm{C}$. The samples were then run at $1.5 \%$ of Agarose gel for their quality check. The sample showing one clear band after PCR samples were sorted and purified with EXO1-SAP (Exonuclease1 and Shrimp Alkaline Phosphatase: USB Corp) with the temperature conditions suggested by manufacturer. The purified PCR amplicons were then ladled with Big Dye Terminator v3.1 (Applied Bio systems) by cycle sequencing, with each side labelled separately. The cycle sequencing PCR reaction contained Ready reaction mix $(2.5 \mathrm{x}) 0.5 \mu \mathrm{L}$, Dilution Buffer $1.75 \mu \mathrm{L}$, Template $(200 \mathrm{ug} / \mu \mathrm{L}) 1 \mu \mathrm{L}$, Primer $(0.8 \mathrm{pMol} / \mu \mathrm{L})$. The cycle sequenced amp icons were then purified with Big Dye (R) X Terminator (TM)(Big Dye Terminator v3.1 clean up Applied Bio systems, USA) each side labelled separately and were sequences on ABI 3130 DNA genetic analyser. $2 \mu \mathrm{L}$, MQ Water $4.75 \mu \mathrm{L}$. The cycle sequencing conditions was Initial Denaturation of $960 \mathrm{C}$ for
$1 \mathrm{~min}$ followed by 35 cycles of Denaturation $960 \mathrm{C}$ for $10 \mathrm{sec}$, annealing $500 \mathrm{C}$ for $5 \mathrm{sec}$, extension $600 \mathrm{C}$ for $4 \mathrm{~min}$ and then the samples were stored at storage temperature of $40 \mathrm{C}$. The sequences were then obtained and analysed in the Sequence Scape software v2.7 for possibilities of indels(Applied Biosystems 3130 Genetic Analyzers).

\section{DATA ANALYSIS}

DNA sequence were also submitted to Gene bank (accession numbers included in appendix online tools). MEGA program (XXX) was used for sequence alignment and further interpretation.

\section{RESULTS}

About 350 GenBank submissions have been made during the entire period of study starting from 2013. There were some interesting observations that are becoming evident from our study that the distribution pattern of species, especially the Schizothorax species, needs to be redefined based on molecular data. More data is also being added so that statistical validation can be carried out.Some of these submissions of various molecular markers are:

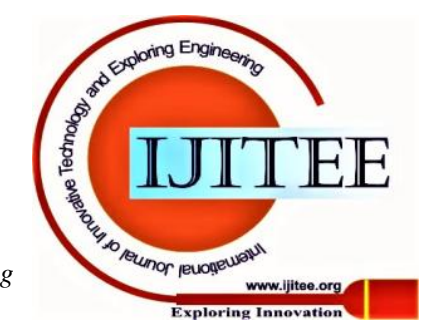


International Journal of Innovative Technology and Exploring Engineering (IJITEE) ISSN: 2278-3075, Volume-8 Issue-10S2, August 2019

\begin{tabular}{|c|c|c|c|}
\hline S.No. & Name of the Species & Voucher No & NCBI Accession No \\
\hline 1 & Bariliusbarna & RS05 & JN965191 \\
\hline 2 & Bariliusbarna & GM01 & JN965190 \\
\hline 3 & Bariliusbendelisis & KR01 & JN965192 \\
\hline 4 & Bariliusbendelisis & KR07 & JN965196 \\
\hline 5 & Bariliusbendelisis & KR06 & JN965195 \\
\hline 6 & Bariliusbendelisis & KR02 & JN965194 \\
\hline 7 & Bariliusbendelisis & GM02 & JN965204 \\
\hline 8 & Bariliusbendelisis & KR04 & JN965212 \\
\hline 9 & Bariliusbendelisis & KR03 & JN965193 \\
\hline 10 & Bariliustileo & GM07 & JN965198 \\
\hline 11 & Bariliustileo & GM08 & JQ692874 \\
\hline 12 & Chaguniuschagunio & GM10 & JN965199 \\
\hline 13 & Garragotyla & BS55 & JN965210 \\
\hline 14 & Garragotyla & HD09 & JN965211 \\
\hline 15 & Garragotyla & HD10 & KC473939 \\
\hline 16 & Garragotylagotyla & RS07 & JN965200 \\
\hline 17 & Macrognathuspancalus & BS123 & KC473940 \\
\hline 18 & Puntiusconchonius & KR10 & JN965201 \\
\hline 19 & Puntiusticto & GM12 & JN965202 \\
\hline 20 & Puntiusticto & GM11 & JN965203 \\
\hline 21 & Schizothorax progastus & UM01 & JN965205 \\
\hline 22 & Schizothorax progastus & UM02 & JQ692872 \\
\hline 23 & Schizothorax progastus & RS01 & JQ692870 \\
\hline 24 & Schizothorax progastus & HD08 & JQ692873 \\
\hline 25 & Schizothorax sp. & HD07 & JQ692871 \\
\hline 26 & Tor chelynoides & UM04 & JN965207 \\
\hline 27 & Tor chelynoides & RS04 & JN965206 \\
\hline 28 & Tor putitora & GM05 & JN965209 \\
\hline 29 & Tor putitora & UM05 & JN965197 \\
\hline 30 & Tor sp. & BSS01 & KC473941 \\
\hline 31 & Tor tor & BS153 & KC473942 \\
\hline 32 & Tor tor & GM06 & JN965208 \\
\hline 33 & Acanthocobitisbotia & GPCR 281AB & KR809714 \\
\hline 34 & Acanthocobitisbotia & GPCR1AB & KU043312 \\
\hline 35 & Acanthocobitisbotia & GPCR2AB & KU043313 \\
\hline 36 & Acanthocobitisbotia & GPCR3AB & KU043314 \\
\hline 37 & Acanthocobitisbotia & GPCR4AB & KU043315 \\
\hline 38 & Badisbadis & GPCR 141BB & KR809715 \\
\hline 39 & Badisbadis & GPCR 282BB & KR809716 \\
\hline 40 & Badisbadis & GPCR 284BB & KR809717 \\
\hline 41 & Badisbadis & GPCR 287BB & KR809718 \\
\hline 42 & Badisbadis & GPCR5BB & KU043316 \\
\hline 43 & Badisbadis & GPCR6BB & KU043317 \\
\hline 44 & Badisbadis & GPCR7BB & KU043318 \\
\hline 45 & Badisbadis & GPCR8BB & KU043319 \\
\hline 46 & Bariliusbarna & GPCR 438BB & KR809719 \\
\hline 47 & Bariliusbarna & GPCR9BB & KU043320 \\
\hline 48 & Bariliusbarna & GPCR10BB & KU043321 \\
\hline 49 & Bariliusbarna & GPCR11BB & KU043322 \\
\hline 50 & Bariliusbarna & GPCR12BB & KU043323 \\
\hline 51 & Bariliusbendelisis & GPCR 113BB & KR809720 \\
\hline 52 & Bariliusbendelisis & GPCR 114BB & KR80 \\
\hline 53 & Bariliusbendelisis & GPCR13BB & KU04 \\
\hline 54 & Bariliusbendelisis & GPCR14BB & KU04 \\
\hline
\end{tabular}


Decoding Evolution of Native Fishes in Garhwal Himalaya using Molecular Markers and DNA Barcoding

\begin{tabular}{|c|c|c|c|}
\hline 55 & Bariliusbendelisis & GPCR15BB & KU043326 \\
\hline 56 & Bariliusvagra & GPCR 112BV & KR809722 \\
\hline 57 & Bariliusvagra & GPCR 115BV & KR809723 \\
\hline 58 & Bariliusvagra & GPCR 270BV & KR809724 \\
\hline 59 & Bariliusvagra & GPCR 272BV & KR809725 \\
\hline 60 & Bariliusvagra & GPCR 273BV & KR809726 \\
\hline 61 & Bariliusvagra & GPCR 274BV & KR809727 \\
\hline 62 & Bariliusvagra & GPCR 385BV & KR809728 \\
\hline 63 & Channagachua & GPCR 142CG & KR809729 \\
\hline 64 & Channagachua & GPCR 16CG & KU043327 \\
\hline 65 & Channagachua & GPCR 17CG & KU043328 \\
\hline 66 & Channagachua & GPCR 18CG & KU043329 \\
\hline 67 & Channagachua & GPCR 19CG & KU043330 \\
\hline 68 & Channapunctata & GPCR 146CP & KR809730 \\
\hline 69 & Channapunctata & GPCR 20CP & KU043331 \\
\hline 70 & Channapunctata & GPCR 21CP & KU043332 \\
\hline 71 & Channapunctata & GPCR 22CP & KU043333 \\
\hline 72 & Channapunctata & GPCR 23CP & KU043334 \\
\hline 73 & Cурrinuscarpio & GPCR 223CC & KR809731 \\
\hline 74 & Cyprinuscarpio & GPCR $225 \mathrm{CC}$ & KR809732 \\
\hline 75 & Cyprinuscarpio & GPCR 293CC & KR809733 \\
\hline 76 & Cyprinuscarpio & GPCR 294CC & KR809734 \\
\hline 77 & Cyprinuscarpio & GPCR 295CC & KR809735 \\
\hline 78 & Cyprinuscarpio & GPCR50CC & KR809736 \\
\hline 79 & Daniodevario & GPCR 236DD & KR809737 \\
\hline 80 & Daniodevario & GPCR 237DD & KR809738 \\
\hline 81 & Daniodevario & GPCR24DD & KU043335 \\
\hline 82 & Daniodevario & GPCR25DD & KU043336 \\
\hline 83 & Daniodevario & GPCR26DD & KU043337 \\
\hline 84 & Garragotyla & GPCR144GG & KR809739 \\
\hline 85 & Garragotyla & GPCR 27GG & KU043338 \\
\hline 86 & Garragotyla & GPCR28GG & KU043339 \\
\hline 87 & Garragotyla & GPCR29GG & KU043340 \\
\hline 88 & Garragotyla & GPCR30GG & KU043341 \\
\hline 89 & Garralamta & GPCR 145GL & KR809740 \\
\hline 90 & Garralamta & GPCR31GL & KU043342 \\
\hline 91 & Garralamta & GPCR32GL & KU043343 \\
\hline 92 & Garralamta & GPCR33GL & KU043344 \\
\hline 93 & Garralamta & GPCR34GL & KU043345 \\
\hline 94 & Lepidocephalichthysguntea & GPCR 280LG & KR809741 \\
\hline 95 & Lepidocephalichthysguntea & GPCR 147LG & KR809742 \\
\hline 96 & Lepidocephalichthysguntea & GPCR35LG & KU043346 \\
\hline 97 & Lepidocephalichthysguntea & GPCR36LG & KU043347 \\
\hline 98 & Lepidocephalichthysguntea & GPCR37LG & KU043348 \\
\hline 99 & Lepidocephalichthys sp. & GPCR 289Lsp. & KR809743 \\
\hline 100 & Lepidocephalichthys sp. & GPCR38Lsp. & KU043349 \\
\hline 101 & Lepidocephalichthys sp. & GPCR39Lsp. & KU043350 \\
\hline 102 & Lepidocephalichthys sp. & GPCR40Lsp. & KU043351 \\
\hline 103 & Lepidocephalichthys sp. & GPCR41Lsp. & KU043352 \\
\hline 104 & Mystusvittatus & GPCR 288MV & KR809744 \\
\hline 105 & Mystusvittatus & GPCR42MV & KU043353 \\
\hline 106 & Mystusvittatus & GPCR43MV & KU043354 \\
\hline 107 & Mystusvittatus & GPCR44MV & KU043355 \\
\hline 108 & Mystusvittatus & GPCR45MV & KU043356 \\
\hline 109 & Nemacheilusmontana & GPCR 58NM & KR809745 \\
\hline 110 & Nemacheilusmontana & GPCR46NM & KU04. \\
\hline 111 & Nemacheilusmontana & GPCR47MV & KU \\
\hline 112 & Nemacheilusmontana & GPCR48MV & KU04 \\
\hline
\end{tabular}




\begin{tabular}{|c|c|c|c|}
\hline 113 & Nemacheilusmontana & GPCR49MV & KU043360 \\
\hline 114 & Pseudecheneissulcata & GPCR 197PS & KR809746 \\
\hline 115 & Pseudecheneissulcata & GPCR 292PS & KR809747 \\
\hline 116 & Pseudecheneissulcata & GPCR 63PS & KR809748 \\
\hline 117 & Pseudecheneissulcata & GPCR50PS & KU043361 \\
\hline 118 & Pseudecheneissulcata & GPCR51PS & KU043362 \\
\hline 119 & Pseudecheneissulcata & GPCR52PS & KU043363 \\
\hline 120 & Puntiuschelynoides & GPCR 196PC & KR809749 \\
\hline 101 & Puntiuschelynoides & GPCR 170PC & KR809750 \\
\hline 122 & Puntiuschelynoides & GPCR 171PC & KR809751 \\
\hline 123 & Puntiuschelynoides & GPCR 172PC & KR809752 \\
\hline 124 & Puntiuschelynoides & GPCR 195PC & KR809753 \\
\hline 125 & Puntiuschelynoides & GPCR 221PC & KR809754 \\
\hline 126 & Puntiuschelynoides & GPCR 262PC & KR809755 \\
\hline 127 & Puntiuschelynoides & GPCR 263PC & KR809756 \\
\hline 128 & Puntiuschelynoides & GPCR 267PC & KR809757 \\
\hline 129 & Puntiuschelynoides & GPCR 331PC & KR809758 \\
\hline 130 & Puntiuschelynoides & GPCR 387PC & KR809759 \\
\hline 131 & Puntiuschelynoides & GPCR 390PC & KR809760 \\
\hline 132 & Puntiuschelynoides & GPCR 430PC & KR809761 \\
\hline 133 & Puntiuschelynoides & GPCR 431PC & KR809762 \\
\hline 134 & Puntiuschelynoides & GPCR 432PC & KR809763 \\
\hline 135 & Salmotrutta & GPCR 121BT & KR809764 \\
\hline 136 & Salmotrutta & GPCR 124BT & KR809765 \\
\hline 137 & Salmotrutta & GPCR 126BT & KR809766 \\
\hline 138 & Salmotrutta & GPCR 128BT & KR809767 \\
\hline 139 & Salmotrutta & GPCR 1BT & KR809768 \\
\hline 140 & Schizothorax plagiostomus & GPCR 101SP & KR809769 \\
\hline 141 & Schizothorax plagiostomus & GPCR53SP & KU043364 \\
\hline 142 & Schizothorax plagiostomus & GPCR54SP & KU043365 \\
\hline 143 & Schizothorax plagiostomus & GPCR55SP & KU043366 \\
\hline 1444 & Schizothorax plagiostomus & GPCR56SP & KU043367 \\
\hline 145 & Schizothorax progastus & GPCR 105SP & KR809770 \\
\hline 146 & Schizothorax progastus & GPCR 162SP & KR809771 \\
\hline 147 & Schizothorax progastus & GPCR 356SP & KR809772 \\
\hline 148 & Schizothorax progastus & GPCR 4SP & KR809773 \\
\hline 149 & Schizothorax progastus & GPCR 9SP & KR809774 \\
\hline 150 & Schizothorax progastus & GPCR 100SP & KR809775 \\
\hline 151 & Schizothorax progastus & GPCR 131SP & KR809776 \\
\hline 152 & Schizothorax progastus & GPCR 160SP & KR809777 \\
\hline 153 & Schizothorax progastus & GPCR 227SP & KR809778 \\
\hline 154 & Schizothorax progastus & GPCR 374SP & KR809779 \\
\hline 155 & Schizothorax progastus & GPCR 97SP & KR809780 \\
\hline 156 & Schizothorax sinuatus & GPCR 110SS & KR809781 \\
\hline 157 & Schizothorax sinuatus & GPCR 111SS & KR809782 \\
\hline 158 & Schizothorax sinuatus & GPCR 57SS & KU043368 \\
\hline 159 & Schizothorax sinuatus & GPCR 58SS & KU043369 \\
\hline 160 & Schizothorax sinuatus & GPCR 59SS & KU043370 \\
\hline 161 & Schizothorax richardsonii & GPCR 1SR & KU695217 \\
\hline 162 & Schizothorax richardsonii & GPCR 2SR & KU695218 \\
\hline 163 & Schizothorax richardsonii & GPCR 3SR & KU695219 \\
\hline 164 & Schizothorax richardsonii & GPCR 4SR & KU695220 \\
\hline 165 & Schizothorax richardsonii & GPCR 5SR & KU695221 \\
\hline 166 & Tor putitora & GPCR 151TP & KR809783 \\
\hline 167 & Tor putitora & GPCR 382TP & KR809784 \\
\hline 168 & Tor putitora & GPCR 383TP & KR809 \\
\hline 169 & Tor putitora & GPCR 384TP & KR8 \\
\hline 170 & Tor putitora & GPCR 51TP & KR8 \\
\hline
\end{tabular}


Acknowledgments Our humble Acknowledgments to the, Graphic Era Deemed to be University, Dehradun for encouragement .

\section{REFERENCES}

1. Adams, C.I.; Knapp, M.; Gemmell, N.J.; Jeunen, G.-J.; Bunce, M.; Lamare, M.D.; Taylor, H.R. (2019) Beyond Biodiversity: Can Environmental DNA (eDNA) Cut It as a Population Genetics Tool? Genes, 10, 192

2. Barat A, et al., (2011). Phylogenetic analysis of fishes of the subfamily Schizothoracinae (Teleostei: Cyprinidae) from Indian Himalayas using cytochrome b gene, Indian J. Fish., 59(1): 43-47.

3. Chandra S et al., (2012). DNA Bar-Coding of Indian Coldwater Fishes of Genus Schizothorax (Family: Cyprinidae) from Western Himalaya, World Journal of Fish and Marine Sciences 4 (4): 430-435, 2012 ISSN 2078-4589 @ IDOSI Publications,

4. Ferguson, A., Taggart, J.B., Prodohl, P.A., McMeel, O., Thompson, C., Stone, C., McGinnity, P. and Hynes, R.A. (1995).The application of molecular markers to the study and conservation of fish populations with special reference to Salmo.Journal of Fish Biology, 47(A), 103-126.

5. Hadrys, H., Balick, M. and Schierwater, B. (1992). Applications of random amplified polymorphic DNA (RAPD) in molecular ecology. Mol. Ecol., 1: 55-63.

6. Hansen, M.M. (2003). Application of molecular markers in population and conservation genetics, with special emphasis on fishes. DSc Thesis, Faculty of Natural Sciences, University of Aarhus, $68 \mathrm{pp}$.

7. Hebert, P. D., Cywinska, A., Ball, S. L., \&Dewaard, J. R. (2003).Biological identifications through DNA barcodes.Proceedings of the Royal Society of London. Series B: Biological Sciences, 270(1512), 313-321.

8. Hebert, P. D., Stoeckle, M. Y., Zemlak, T. S., \& Francis, C. M. (2004).Identification of birds through DNA barcodes.PLoS biology, 2(10).

9. Krehenwinkel, H.; Pomerantz, A.; Prost, S. (2019) Genetic Biomonitoring and Biodiversity Assessment Using Portable Sequencing Technologies: Current Uses and Future Directions.Genes, 10,858 .

10. Magoulas, A. (1998). Application of molecular markers to aquaculture and broodstock management with special emphasis on microsatellite DNA. Cahiers Options Mediterrannes, 34: 153- 168

11. McConnell, S.K., O'Reilly, P., Hamilton, L., Wright, J.N. and Bentzen, P. (1995).Polymorphic microsatellite loci from Atlantic salmon (Salmosalar) - genetic differentiation of North American and European populations.Can. J. Fish.Aquat.Sci., 52: 1863- 1872.

12. Parker, Patricia G. et al., (1998). "What Molecules Can Tell Us about Populations: Choosing and Using a Molecular Marker". Ecology 79 (2): 361-382.

13. Petzold, A., \&Hassanin, A. (2020). A comparative approach for species delimitation based on multiple methods of multi-locus DNA sequence analysis: A case study of the genus Giraffa (Mammalia, Cetartiodactyla). PloS one, 15(2), e0217956.

14. Siozios, S., Massa, A., Parr, C. L., Verspoor, R. L., \& Hurst, G. D. (2020). DNA barcoding reveals incorrect labelling of insects sold as food in the UK. PeerJ, 8, e8496.

15. Thapliyal M., Sati B.K., Kumar R., Chandra T., Thapliyal A., (2013).Molecular taxonomy of fresh water fishes from song river Uttarakhand using mitochondrial cytochrome-c oxidase-1 gene, Environment Conservation Journal, 14(3).

16. Xing, B., Zhang, Z., Sun, R., Wang, Y., Lin, M., \& Wang, C. (2020). Mini-DNA barcoding for the identification of commercial fish sold in the markets along the Taiwan Strait. Food Control, 107143. 